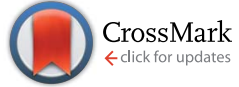

Cite this: RSC Adv., 2017, 7, 6966

Received 18th October 2016

Accepted 2nd January 2017

DOI: $10.1039 / c 6 r a 25429 b$

www.rsc.org/advances

\section{Efficient access to triarylmethanes through decarboxylation $\uparrow$}

\author{
Tiash Saha, ${ }^{a}$ M. Srinivas Lavanya Kumar, ${ }^{a}$ Saurav Bera, ${ }^{a}$ Bidhu Bhusan Karkara ${ }^{a}$ \\ and Gautam Panda*ab
}

A new synthetic approach has been developed for efficient access to triarylmethanes by palladium catalyzed decarboxylative cross coupling reactions. The reaction features $\mathrm{sp}^{2}-\mathrm{sp}^{3}$ coupling where benzoic acids upon decarboxylation reacted with diaryl methyl iodides having both electron donating and withdrawing functionalities, leading to the synthesis of diverse triarylmethanes.

\section{Introduction}

The triarylmethane class of molecules contains several biologically significant motifs displaying a wide range of pharmaceutical profiles. ${ }^{1}$ It forms the basic skeleton of several antitubercular, ${ }^{2 a}$ anticancer, ${ }^{2 b, c}$ antidiabetic ${ }^{2 d}$ and other medicinally important molecules, Fig. 1 . These privileged architectures can be diversely

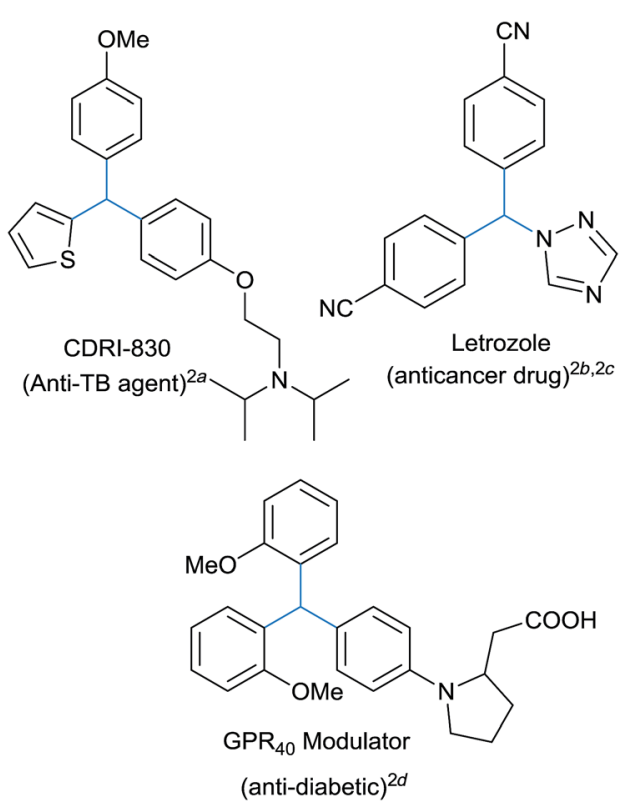

Fig. 1 Representative triarylmethane containing architectures.

\footnotetext{
${ }^{a}$ Medicinal and Process Chemistry Division, CSIR-Central Drug Research Institute, BS 10/1, Jankipuram Extension, Sitapur Road, Lucknow 226031, India. E-mail: gautam. panda@gmail.com; gautam_panda@cdri.res.in; Fax: +91-522-2771941; Tel: +91522-2772450 ext. 4661; +91-522-2772550 ext. 4662

${ }^{b}$ Academy of Scientific and Innovative Research, New Delhi 110001, India

$\dagger$ Electronic supplementary information (ESI) available. See DOI: $10.1039 / \mathrm{c} 6 \mathrm{ra} 25429 \mathrm{~b}$
}

functionalized in the quest for new therapeutic agents and unexplored biologically relevant chemical spaces. ${ }^{3}$ Several triarylmethane based dyes were also developed as sensitizers, exhibiting selective phototoxicity towards cancerous cell lines and thus forms the basic principle of photodynamic therapy. ${ }^{4}$ These triarylmethanes exhibit interesting properties like photo lability, photo ionization which were successfully exploited for the development of many organic based dyes, photochromatic devices and fluorescent probes. ${ }^{4 b, 5}$ Apart from this, these class of compounds often found their explosive growth in the construction of supramolecular architectures. ${ }^{6}$

Conventionally, triarylmethanes are synthesized through typical Friedel-Crafts alkylation of diarylcarbinols with aromatic ring as nucleophile ${ }^{7}$ or hydroarylation of arenes with aromatic aldehydes or imines in the presence of either suitable Bronsted or Lewis acid systems. ${ }^{1 a}$ Recently, Crudden et al. reported the synthesis of triarylmethanes through arylative desulfonation ${ }^{8}$ whereas Chakravarty et al. reported Friedel-Crafts reaction on benzylic phosphates for the synthesis of triarylmethanes. ${ }^{9 a}$ These procedures often suffer from lack of selectivity, involves multistep synthesis and moreover is limited to only electron rich arene systems. ${ }^{9 b, c}$

Due to this, cross coupling based strategies emerged as promising avenue for the short and efficient synthesis of triarylmethanes. ${ }^{10}$ Oshima et al. reported palladium catalyzed direct $\mathrm{C}-\mathrm{H}$ bond activation ${ }^{11}$ of aryl(azaaryl)methanes for synthesis of triarylmethanes. L. A. López et al. reported zinc catalyzed cascade reaction between enynones and azoles resulting in the diversely substituted triarylmethanes synthesis. ${ }^{12} \mathrm{~A}$ reductive cross coupling was developed by Zhang et al. using benzophenone tosylhydrazones using palladium catalyst. ${ }^{13}$ Jarvo et al. synthesized enantioenriched triarylmethanes by stereospecific cross coupling in presence of nickel catalyst. ${ }^{14}$ Similarly, palladium catalysed C$\mathrm{H} / \mathrm{C}-\mathrm{O}$ coupling of oxazoles with diarylmethanol derivatives have also been reported for synthesis of triarylmethanes. ${ }^{15}$

Decarboxylative cross coupling reaction has been emerged as a powerful strategy for $\mathrm{C}-\mathrm{C}$ bond formation ${ }^{16}$ in the recent past 
and had been largely explored. Organometallic species formed via expulsion of $\mathrm{CO}_{2}$ from commercially available arene carboxylic acids were ventured in this cross coupling strategy. Goossen et al. synthesized arylalkenes via decarboxylative cross coupling. ${ }^{17}$ Palladium catalyzed decarboxylative cross coupling, as demonstrated by Becht and coworkers ${ }^{\mathbf{1 8}}$ were used in the synthesis of biaryl motifs. Even industrial scale production of commercially important biaryls like intermediates of agrochemicals boscalid, bixafen has been demonstrated by this strategy. ${ }^{19}$ Decarboxylation reactions were well explored in $\mathrm{sp}-$ $\mathrm{sp}^{2}, \mathrm{sp}^{2}-\mathrm{sp}^{2}$ cross couplings whereas $\mathrm{sp}^{2}-\mathrm{sp}^{3}$ coupling was hardly explored. ${ }^{20}$ In our quest for highly desirable triarylmethanes, ${ }^{1 \boldsymbol{b}, 2 \boldsymbol{b}, \boldsymbol{7}}$ we here reported an efficient palladium catalyzed decarboxylative $\mathrm{sp}^{2}-\mathrm{sp}^{3}$ cross coupling for the access to triarylmethanes with both electron demanding and donating substitutions using commercially available benzoic acids with tailor made diarylmethyl iodides.

\section{Results and discussion}

In principle, we presumed that aromatic carboxylic acids ${ }^{\mathbf{1 6 a}}$ can be used as boronic acid surrogate and palladium catalyst could synchronize coupling ${ }^{\mathbf{1 6} b}$ between decarboxylated benzoic species with diarylmethyl iodides leading to these valuable architectures. For the test reaction, we have chosen 2,6 dimethoxy benzoic acid and diphenylmethyl iodide ${ }^{21}$ as model substrates. Preliminary screening using $\mathrm{Pd}(\mathrm{OAc})_{2}$ and dppp as a ligand in the presence of $\mathrm{Ag}_{2} \mathrm{CO}_{3}$ (Table 1, entries 1 and 2) was unsuccessful. Then changing the palladium catalyst to $\mathrm{Pd}_{2}(\mathrm{dba})_{3}$ (Table 1 , entry 3 ) resulted in $22 \%$ yield. Altering the bases to $\mathrm{Cs}_{2} \mathrm{CO}_{3}, \mathrm{Li}_{2} \mathrm{CO}_{3}, \mathrm{Cs}_{2} \mathrm{CO}_{3}$, AgOAc, DBU and $\mathrm{K}_{2} \mathrm{CO}_{3}$ were unable to increase the yield of the desired product to significant level. Employing additive such as CuI, resulted in trace amount of product. Keeping palladium source intact, varying the ligands to triphenyl phosphine and 1,10-phenanthroline resulted in more or less comparable yields but xantphos gave promising yields (Table 1, entries 4-6). Some by-products due to protodecarboxylation of carboxylic acids and deiodination of diphenyl methyl iodides were also isolated. ${ }^{22,23}$ Then surveying various palladium catalysts like $\mathrm{Pd}\left(\mathrm{CH}_{3} \mathrm{CN}\right)_{2} \mathrm{Cl}_{2}$ and $\mathrm{Pd}(\mathrm{Cl})_{2}(-$ $\left.\mathrm{PPh}_{3}\right)_{2}, \mathrm{Pd}(\text { acac })_{2}, \mathrm{PdCl}_{2}$ and careful solvent screening revealed that $\mathrm{PdCl}_{2}$ along with xantphos ligand, $\mathrm{Ag}_{2} \mathrm{CO}_{3}$ as base in presence of DMSO offered the ideal conditions for the reaction (Table 1, entry 11).

After optimizing the catalyst system for decarboxylative coupling reactions, the scopes of substrates were evaluated with reference to both the coupling partners (Table 2). Poor yields were obtained in the case of diphenylmethyl bromide (38\%) and

Table 1 Optimization of conditions for decarboxylative cross coupling

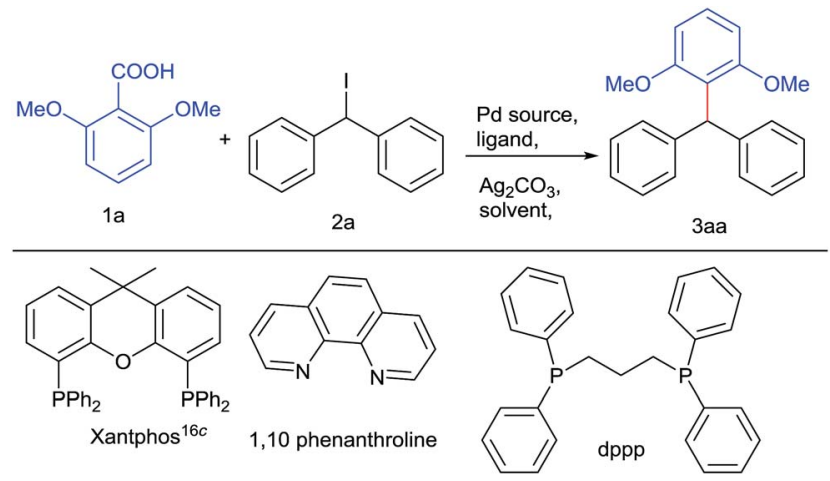

\begin{tabular}{|c|c|c|c|c|}
\hline Entry $^{a}$ & Catalyst & Ligand & Solvent & Yield $^{f}(\%)$ \\
\hline $\mathbf{1}^{b}$ & $\mathrm{Pd}(\mathrm{OAc})_{2}$ & Dppp & THF & 0 \\
\hline 2 & $\mathrm{Pd}(\mathrm{OAc})_{2}$ & Dppp & DMF & $\mathrm{Nd}^{g}$ \\
\hline 4 & $\mathrm{Pd}_{2}(\mathrm{dba})_{3}$ & 1,10-Phenanthroline & DMF & $\mathrm{Nd}^{g}$ \\
\hline 5 & $\mathrm{Pd}_{2}(\mathrm{dba})_{3}$ & Xantphos & DMF & 42 \\
\hline 6 & $\mathrm{Pd}_{2}(\mathrm{dba})_{3}$ & $\mathrm{PPh}_{3}$ & DMF & 34 \\
\hline 9 & $\mathrm{Pd}(\mathrm{Cl})_{2}\left(\mathrm{PPh}_{3}\right)_{2}$ & Xantphos & DMF & 40 \\
\hline 10 & $\mathrm{Pd}(\mathrm{OAc})_{2}$ & Xantphos & $\mathrm{DMF}$ & 42 \\
\hline 11 & $\mathbf{P d C l}_{2}$ & Xantphos & DMSO & $62(68)^{e}$ \\
\hline 12 & $\mathrm{PdCl}_{2}$ & Xantphos & NMP : mesitylene (1: 1) & Trace \\
\hline 13 & $\mathrm{PdCl}_{2}$ & Xantphos & Diglyme & 15 \\
\hline
\end{tabular}

${ }^{a}$ Reaction conditions: $\mathrm{Pd}$ source (0.1 equiv.), ligand (0.2 equiv.), $\mathrm{Ag}_{2} \mathrm{CO}_{3}$ (1.5 equiv.), arene carboxylic acid (1.2 equiv.), methyl iodide (1.0 equiv.), $100{ }^{\circ} \mathrm{C} .{ }^{b}$ Reaction was performed at $65{ }^{\circ} \mathrm{C}$. ${ }^{c} \mathrm{CuI}$ additive $(0.2$ eq. $)$ is used. ${ }^{d}$ Pd catalyst ( 0.2 eq.) was used. ${ }^{e}$ Reaction was performed at $120{ }^{\circ} \mathrm{C}$. ${ }^{f}$ Overall isolated yield. ${ }^{g} \mathrm{Nd}$ means not determined. 
Table 2 Substrate scope for decarboxylative cross coupling of aromatic carboxylic acid

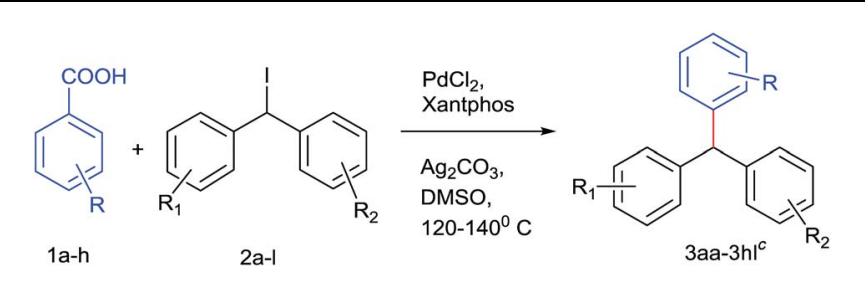

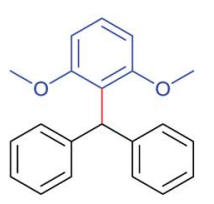
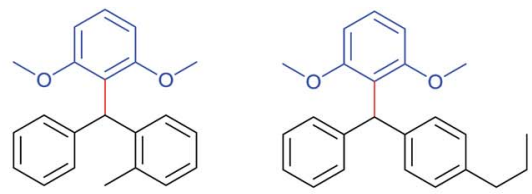

3aa $(68 \%)^{a}$ $18 \mathrm{hrs}$

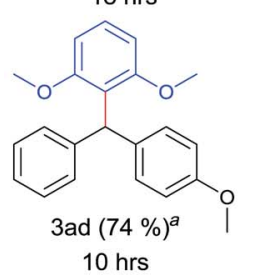
$20 \mathrm{hrs}$
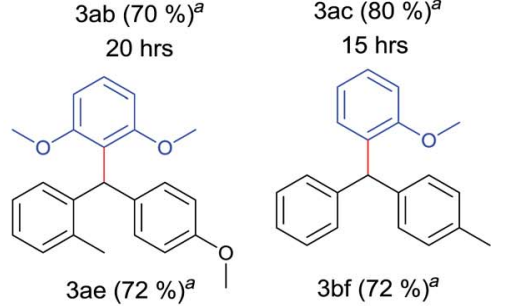<smiles>CCOc1ccc(C(c2ccccc2)c2ccccc2OC)cc1</smiles>

$18 \mathrm{hrs}$

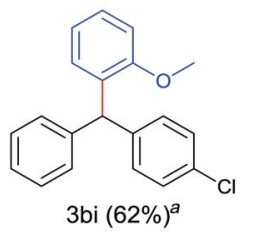

$22 \mathrm{hrs}$<smiles>O=[N+]([O-])c1ccc(F)c(C(c2ccccc2)c2ccc(-c3ccccc3)cc2)c1</smiles>
$3 \mathrm{dk}(42 \%)^{b}$ 29 hrs

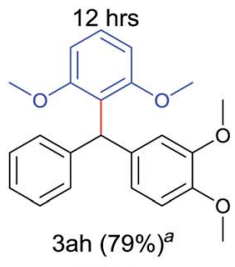

$10 \mathrm{hrs}$
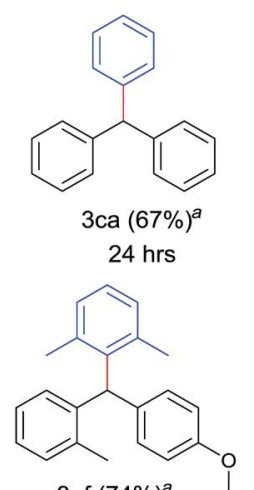

3ef $(74 \%)^{a}$
$3 b f(72 \%)^{a}$

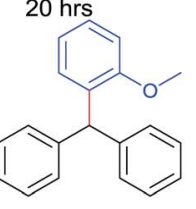

3 ba $(71 \%)^{a}$

$18 \mathrm{hrs}$

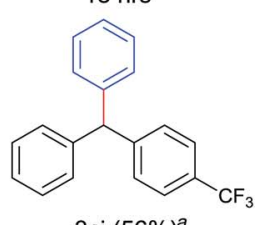

$3 c j(56 \%)^{a}$ 32 hrs

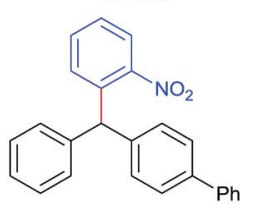

3 fk $(49 \%)^{a}$ $27 \mathrm{hrs}$
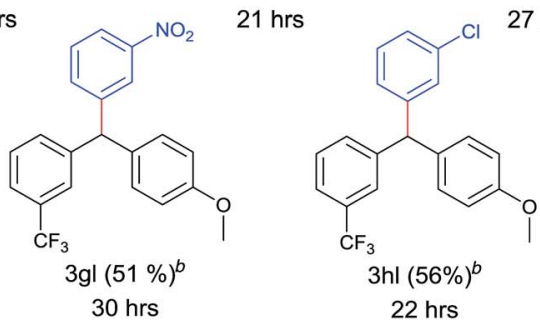

${ }^{a}$ Reaction conditions: substituted diphenylmethyl iodide (1 equiv.), aryl carboxylic acid (1 equiv.), $\mathrm{PdCl}_{2}$ ( 0.1 equiv.), xantphos ( 0.2 equiv.), $\mathrm{Ag}_{2} \mathrm{CO}_{3}$ (1.5 equiv.), DMSO. ${ }^{b}$ Substituted diphenylmethyl iodide (1 equiv.), aryl carboxylic acid (1 equiv.), $\mathrm{PdCl}_{2}$ ( 0.2 equiv.), xantphos (0.4 equiv.), $\mathrm{Ag}_{2} \mathrm{CO}_{3}$ (1.5 equiv), DMSO : DMF (90:10). ${ }^{c}$ Isolated yields after column chromatography in silica gel.
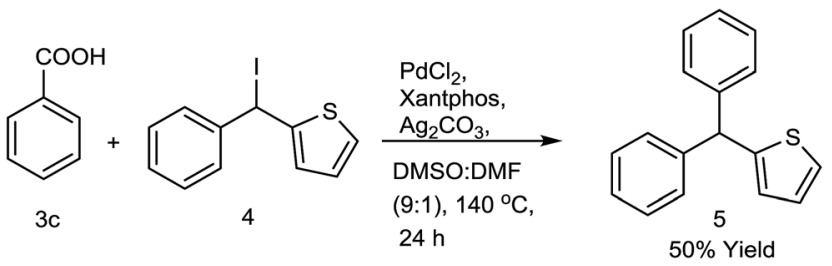

Fig. 2 Synthesis of heteroaryl substituted triarylmethanes.

reaction failed when performed with diphenylmethyl chloride. The electron rich 2,6 dimethoxy benzoic acid 1a was successfully coupled with various diphenylmethyl iodides which provided sterically hindered triarylmethanes $\mathbf{3 a a}-\mathbf{3 a e}, \mathbf{3 a h}$ in good yields. Similarly, 2-methoxy benzoic acid $\mathbf{1 b}$ was successfully cross coupled with diphenylmethyl iodide derivatives 2a, $2 \mathbf{f}, 2 \mathrm{~g}$ and even with the substrate $2 \mathbf{i}$ resulting in triarylmethane derivatives $\mathbf{3 b a}, \mathbf{3 b f}, \mathbf{3 b g}$ and $\mathbf{3 b i}$. Thus, the reaction was also compatible with unsubstituted benzene carboxylic acids affording the products $3 \mathrm{ca}$, $3 \mathrm{cj}$ in $67 \%$ and $56 \%$ yield, respectively. Even sterically demanding benzoic acid 1e also worked well in this strategy leading to cross coupled product 3ef. In order to explore the feasibility of this reaction further, cross coupling reaction was performed between electron poor counterparts. Slight modification in the reaction conditions needed for the elaboration of this cross coupling reaction to electron poor triaryl methanes. Doubling the amount of catalyst and ligand and changing the solvent system to DMSO :DMF (90:10) resulted the coupling of 2-fluoro 5-nitro benzoic acid 1d and 2-nitrobenzoic acid 1f with the methyl iodide 2k successfully in moderate yields leading to $\mathbf{3 d k}$ and $\mathbf{3 f k}$ respectively.

Electron density on aromatic backbone showed strong influence on reactivity and yields. Thus, the catalyst system showed its suitability for wide variety of diphenylmethyl iodides and both electron rich and electron poor benzoic acids successfully underwent decarboxylation to yield diverse triarylmethanes.

After establishing the decarboxylative cross coupling reaction with arenes, we verified the methodology towards synthesizing heteroaryl containing triarylmethanes. Attempted decarboxylative cross coupling (Fig. 2) between phenyl thiophenyl methyl iodide and benzoic acid was also successful using 0.2 equiv. $\mathrm{PdCl}_{2}, 0.4$ equiv. xantphos, 2.0 equiv. $\mathrm{Ag}_{2} \mathrm{CO}_{3}$ at $140{ }^{\circ} \mathrm{C}$ in DMSO : DMF (90:10) for $24 \mathrm{~h}$. Agreeably, the cross coupling product 5 was observed in good yield and it provided a new horizon in the synthesis of antitubercular analogue of CDRI-830 using $\mathrm{sp}^{2}-\mathrm{sp}^{3}$ decarboxylative cross coupling. ${ }^{2 a}$

\section{Experimental section}

\section{General}

All the reactions were carried out in oven-dried glassware under argon atmosphere. Carboxylic acids were purchased from Sigma Aldrich. Palladium catalysts were purchased from Sigma Aldrich. Phosphine ligands were bought from Sigma-Aldrich or Alfa Aesar and used as such. DMSO and DMF was dried according to standard procedure and stored on molecular sieves $4 \AA$ A. All the 
other reagents and solvents mentioned in this text were bought from Sigma Aldrich or Alfa Aesar or spectrochem and purified if necessary. NMR spectra were recorded on 300,400 or $500 \mathrm{MHz}$ spectrometer for ${ }^{1} \mathrm{H}$ NMR, 75 or 100 or $125 \mathrm{MHz}$ for ${ }^{13} \mathrm{C}$ NMR spectroscopy. Chemical shifts are reported in parts per million $(\delta)$ downfield relative to the residual signals of tetramethylsilane in $\mathrm{CDCl}_{3}$. Spin multiplicities are indicated by the following symbol: s (singlet), d (doublet), t (triplet) and m (multiplet).

\section{General procedure for synthesis of diphenyl carbinols}

A $100 \mathrm{ml}$ double-necked round bottom flask was charged with magnesium ( 2 equiv.), pinch of iodine and after flushing with nitrogen, dried THF was added and stirred. Then corresponding bromoarene (1.5 equiv.) was added, the colour of iodine disappeared slowly on time, indicating the generation of Grignard reagent. It was stirred for one hour followed by addition of corresponding carbaldehye ( 1 equiv.). Completion of reaction was determined by TLC. Reaction was quenched by saturated $\mathrm{NH}_{4} \mathrm{Cl}$ solution. Organic layer was extracted thrice with ethyl acetate and concentrated in vacuo. Compound was purified by column chromatography.

\section{General procedure for synthesis of diphenyl methyl iodide}

To oven dried round bottom flask under nitrogen, the alcohol (1 equiv.) and KI (1 equiv.) were added and dissolved in dry 1,4dioxane and stirred for $5 \mathrm{~min}$. Then $\mathrm{BF}_{3} \cdot \mathrm{Et}_{2} \mathrm{O}$ ( 1 equiv.) was added and stirred at room temperature. Completion of reaction was monitored by TLC. The reaction mixture was poured in cold water and extracted with dichloromethane. The organic layer was washed with water and dried by sodium sulphate; solvent was evaporated under reduced pressure. The crude was purified by quickly passing through short pad of silica using hexane/ ethyl acetate $(8: 2)$ as eluent to obtain diphenylmethyl iodides and should be used immediately.

General procedure A for synthesis of various substituted triarylmethanes by decarboxylative cross coupling

To the oven dried round bottom flask was charged with aryl carboxylic acid (1 equiv.), $\mathrm{Ag}_{2} \mathrm{CO}_{3}$ (1.5 equiv.), $\mathrm{PdCl}_{2}$ (0.1 equiv.), xantphos ( 0.2 equiv.), diphenyl methyl iodides ( 1 equiv.) were dissolved in DMSO. The reaction mixture was degassed with argon thrice and stirred in preheated oil bath. Reaction as monitored by TLC after completion, the reaction mixture was cooled to room temperature and filtered through celite bed. The organic layer was washed with saturated $\mathrm{NH}_{4} \mathrm{Cl}$ solution and dried on sodium sulphate and concentrated under reduced pressure. It was later purified by column chromatography on silica gel to obtain corresponding triarylmethanes.

\section{General procedure B for synthesis of substituted triarylmethanes by decarboxylative cross coupling}

To the oven dried round bottom flask was charged with aryl carboxylic acid (1 equiv.), $\mathrm{Ag}_{2} \mathrm{CO}_{3}$ (1.5 equiv.), $\mathrm{PdCl}_{2}$ (0.2 equiv.), xantphos ( 0.4 equiv.), diphenyl methyl iodides ( 1 equiv.) were dissolved in DMSO : DMF $90: 10$. The reaction mixture was degassed with argon thrice and stirred in preheated oil bath at requisite temperature and was monitored by TLC. After the completion of reaction, mixture was cooled to room temperature and filtered through a bed celite with ethyl acetate. The organic layer was washed with saturated $\mathrm{NH}_{4} \mathrm{Cl}$ solution and dried on sodium sulphate and concentrated in vacuo. It was later purified by column chromatography on silica gel to obtain corresponding triarylmethanes.

\section{Spectroscopic data of synthesized compounds}

((2,6-Dimethoxyphenyl)methylene)dibenzene

(3aa).

Prepared following the general procedure A. Rf: 0.35 (2\% EtOAc in Hex). Isolated as pale brown, oily liquid (yield: 68\%). ${ }^{1} \mathrm{H}$ NMR $\left(400 \mathrm{MHz}, \mathrm{CDCl}_{3}\right): \delta$ 7.27-7.06 $(\mathrm{m}, 10 \mathrm{H}), 6.73-6.71(\mathrm{~m}, 1 \mathrm{H})$, 6.46-6.37 (m, 2H), $5.82(\mathrm{~s}, 1 \mathrm{H}), 3.78(\mathrm{~s}, 3 \mathrm{H}), 3.68(\mathrm{~s}, 3 \mathrm{H}) \mathrm{ppm} .{ }^{13} \mathrm{C}$ NMR (100 MHz, $\mathrm{CDCl}_{3}$ ): 158.2 (2C), 143.0 (2C), 129.5 (4C), 128.1 (4C), 126.8 (2C), 124.6, 102.5 (2C), 97.4, 54.3, 54.0, 47.9 ppm. IR (film, $\mathrm{cm}^{-1}$ ): 2817, 1726, 1582, 1231, 814, 770, 637. MS (ESI): $\mathrm{m} / \mathrm{z}$ $305(\mathrm{M}+\mathrm{H})^{+}$. HRMS (ESI): $m / z$ calcd for $\mathrm{C}_{21} \mathrm{H}_{21} \mathrm{O}_{2}[\mathrm{M}+\mathrm{H}]^{+}$ 305.1536, found 305.1542.

1,3-Dimethoxy-2-(phenyl(o-tolyl)methyl)benzene

(3ab). Prepared following the general procedure A. Rf: 0.4 (2\% EtOAc in Hex). Isolated as yellow oily liquid (yield: 70\%). ${ }^{1} \mathrm{H}$ NMR (400 $\mathrm{MHz} \mathrm{CDCl}_{3}$ ): $\delta$ 7.22-7.02 (m, 8H), 6.79-6.35 (m, 4H), $5.89(\mathrm{~s}$, $1 \mathrm{H}), 3.78(\mathrm{~s}, 3 \mathrm{H}), 3.68(\mathrm{~s}, 3 \mathrm{H}), 2.18(\mathrm{~s}, 3 \mathrm{H}) \mathrm{ppm} .{ }^{13} \mathrm{C}$ NMR $(100$ $\left.\mathrm{MHz}, \mathrm{CDCl}_{3}\right)$ : 159.3, 158.0, 143.6, 142.6, 136.7, 130.6, 130.1, 129.5 (2C), 128.9, 128.0 (2C), 126.0, 125.8, 125.4, 124.7, 103.7, 98.6, 55.6, 55.2, 45.9, 19.6 ppm. IR (film, $\mathrm{cm}^{-1}$ ): 2828, 1631, 1536, 1483, 1284, 1171, 823, 772, 656. MS (ESI): $m / z 319(\mathrm{M}+\mathrm{H})^{+}$. HRMS (ESI): $m / z$ calcd for $\mathrm{C}_{22} \mathrm{H}_{23} \mathrm{O}_{2}[\mathrm{M}+\mathrm{H}]^{+} 319.1693$, found 319.1691.

1-Methoxy-2-(phenyl(p-tolyl)methyl)benzene (3bf). Prepared following the general procedure A. Rf: 0.6 (1.5\% EtOAc in Hex). Yield: $72 \% .{ }^{1} \mathrm{H}$ NMR (400 MHz, $\left.\mathrm{CDCl}_{3}\right): \delta 7.23-6.80(\mathrm{~m}, 11 \mathrm{H})$, 6.43-6.34 (m, 2H), 5.69 (s, 1H), $3.70(\mathrm{~s}, 3 \mathrm{H}), 2.27$ (s, 3H) ppm. ${ }^{13} \mathrm{C}$ NMR (100 MHz, $\mathrm{CDCl}_{3}$ ): 156.2, 144.4, 141.1, 141.0, 135.1, 135.0, $132.7,132.6$, 129.1, 129.0, 129.0, 128.6, 127.8, 125.6, 125.5, 124.1, 103.7, 98.6, 55.8, 48.6, 21.0 ppm. IR (film, $\mathrm{cm}^{-1}$ ): 1689, 1276, 948, 879, 847, 716. MS (ESI): $m / z 289(\mathrm{M}+\mathrm{H})^{+}$. HRMS (ESI): $m / z$ calcd for $\mathrm{C}_{21} \mathrm{H}_{21} \mathrm{O}[\mathrm{M}+\mathrm{H}]^{+} 289.1587$, found 289.1594.

1,3-Dimethoxy-2-(phenyl(4-propylphenyl)methyl)benzene (3ac). Prepared following the general procedure A. Rf: 0.3 (2\% EtOAc in Hex). Yield: $80 \%,{ }^{1} \mathrm{H}$ NMR (400 MHz, $\left.\mathrm{CDCl}_{3}\right): \delta 7.25-6.96(\mathrm{~m}, 9 \mathrm{H})$, 6.75-6.73 (m, 1H), 6.45-6.37 (m, 2H), $5.79(\mathrm{~s}, 1 \mathrm{H}), 3.77$ (s, 3H), 3.68 $(\mathrm{s}, 3 \mathrm{H}), 2.54(\mathrm{t}, 2 \mathrm{H}, J=7.83 \mathrm{~Hz}), 1.64-1.59(\mathrm{~m}, 2 \mathrm{H}), 0.92(\mathrm{t}, 3 \mathrm{H}, J=$ $7.32 \mathrm{~Hz}$ ) ppm. ${ }^{13} \mathrm{C} \mathrm{NMR}\left(100 \mathrm{MHz}, \mathrm{CDCl}_{3}\right): 159.3,157.9,144.5$, 141.3, 140.1, 130.7, 129.3 (2C), 129.1 (2C), 128.1 (2C), 128.0 (2C), 125.8, 125.5, 103.7, 98.6, 55.5, 55.2, 48.6, 37.6, 24.4, 13.9 ppm. IR (film, $\mathrm{cm}^{-1}$ ): 3248, 1692, 1452, 1268, 910, 880, 730, 633. MS (ESI): $m / z 347(\mathrm{M}+\mathrm{H})^{+}$. HRMS (ESI): $m / z$ calcd for $\mathrm{C}_{24} \mathrm{H}_{27} \mathrm{O}_{2}(\mathrm{M}+\mathrm{H})^{+}$ 347.2006, found 347.2003.

1,3-Dimethoxy-2-((4-methoxyphenyl)(phenyl)methyl)benzene (3ad). Prepared following the general procedure A. Rf: $0.2(3 \%$ EtOAc in Hex). Yield: $74 \%,{ }^{1} \mathrm{H}$ NMR (400 $\left.\mathrm{MHz} \mathrm{CDCl}_{3}\right): \delta 7.35-$ $6.36(\mathrm{~m}, 12 \mathrm{H}), 5.76(\mathrm{~s}, 1 \mathrm{H}), 3.76(\mathrm{~s}, 3 \mathrm{H}), 3.76(\mathrm{~s}, 3 \mathrm{H}), 3.67(\mathrm{~s}$, $3 \mathrm{H}) \mathrm{ppm} .{ }^{13} \mathrm{C} \mathrm{NMR}\left(100 \mathrm{MHz}, \mathrm{CDCl}_{3}\right): 159.3,158.9,157.9,144.6$, 
136.3, 130.6, 130.3, 129.3, 128.5, 128.3, 128.0, 127.1, 127.0, 125.8, 125.5, 113.4, 103.7, 98.6, 55.6, 55.2, 55.2, 48.3 ppm. IR (film, $\mathrm{cm}^{-1}$ ): 2802, 1664, 1596, 1327, 932, 815, 804, 730, 623. MS (ESI): $m / z 335(\mathrm{M}+\mathrm{H})^{+}$. HRMS (ESI): $m / z$ calcd for $\mathrm{C}_{22} \mathrm{H}_{23} \mathrm{O}_{3}(\mathrm{M}+\mathrm{H})^{+}$ 335.1642, found 335.1632.

1,3-Dimethoxy-2-((4-methoxyphenyl)(o-tolyl)methyl)benzene (3ae). Prepared following the general procedure A. Rf: 0.3 (4\% EtOAc in Hex). Yield: $72 \%,{ }^{1} \mathrm{H}$ NMR (400 $\left.\mathrm{MHz} \mathrm{CDCl}_{3}\right): \delta 7.17-$ $6.28(\mathrm{~m}, 11 \mathrm{H}), 5.76(\mathrm{~s}, 1 \mathrm{H}), 3.70(\mathrm{~s}, 6 \mathrm{H}), 3.60(\mathrm{~s}, 3 \mathrm{H}), 2.10(\mathrm{~s}$, $3 \mathrm{H}) \mathrm{ppm} .{ }^{13} \mathrm{C} \mathrm{NMR}\left(100 \mathrm{MHz}, \mathrm{CDCl}_{3}\right): 159.3,157.9,157.7,143.0$, 136.6, 135.6, 130.5, 130.3, 130.1 (2C), 128.7 (2C), 125.9, 125.4, 125.0, 113.4, 103.7, 98.6, 55.6, 55.2, 55.1, 45.1, 19.5 ppm. IR (film, $\mathrm{cm}^{-1}$ ): 2902, 1685, 1536, 1347, 911, 776. MS (ESI): $\mathrm{m} / z 349$ $(\mathrm{M}+\mathrm{H})^{+}$. HRMS (ESI): $m / z$ calcd for $\mathrm{C}_{23} \mathrm{H}_{25} \mathrm{O}_{3}(\mathrm{M}+\mathrm{H})^{+} 349.1798$, found 349.1810 .

1-((4-Ethoxyphenyl)(phenyl)methyl)-2-methoxybenzene (3bg). Prepared following the general procedure A. Rf: 0.3 (2\% EtOAc in Hex). Yield: $68 \%,{ }^{1} \mathrm{H}$ NMR $\left(400 \mathrm{MHz}, \mathrm{CDCl}_{3}\right): \delta 7.08-6.29(\mathrm{~m}, 13 \mathrm{H})$, $5.62(\mathrm{~s}, 1 \mathrm{H}), 3.90(\mathrm{~m}, 2 \mathrm{H}), 3.62(\mathrm{~s}, 3 \mathrm{H}), 1.31(\mathrm{t}, 3 \mathrm{H}, J=5.4 \mathrm{~Hz}) \mathrm{ppm}$. ${ }^{13} \mathrm{C} \mathrm{NMR}\left(100 \mathrm{MHz}, \mathrm{CDCl}_{3}\right): 157.0,156.2,144.6,136.2,136.1,132.6$, 132.5, 130.0 (2C), 129.1 (3C), 127.9 (2C), 125.6, 124.3, 113.9, 95.5, 63.3, 55.8, 48.2, 14.9 ppm. IR (film, $\mathrm{cm}^{-1}$ ): 2815, 1664, 1592, 1585, 1320, 1104, 932, 812, 730, 623. MS (ESI): $m / z 319(\mathrm{M}+\mathrm{H})^{+}$. HRMS (ESI): $m / z$ calcd for $\mathrm{C}_{22} \mathrm{H}_{23} \mathrm{O}_{2}[\mathrm{M}+\mathrm{H}]^{+} 319.1693$, found 319.1693.

2-((3,4-Dimethoxyphenyl)(phenyl)methyl)-1,3-dimethoxybenzene (3ah). Prepared following the general procedure A. Rf: 0.3 (5\% EtOAc in Hex). Yield: $79 \%,{ }^{1} \mathrm{H}$ NMR (400 $\mathrm{MHz}, \mathrm{CDCl}_{3}$ ): $\delta 7.18-6.29(\mathrm{~m}, 11 \mathrm{H}), 5.68(\mathrm{~s}, 1 \mathrm{H}), 3.76(\mathrm{~s}, 3 \mathrm{H}), 3.70(\mathrm{~s}, 3 \mathrm{H}), 3.67$ $(\mathrm{s}, 3 \mathrm{H}), 3.61(\mathrm{~s}, 3 \mathrm{H}) \mathrm{ppm} .{ }^{13} \mathrm{C} \mathrm{NMR}\left(100 \mathrm{MHz}, \mathrm{CDCl}_{3}\right): 158.2$, 156.8, 147.4, 146.0, 143.3, 135.6, 129.4 (2C), 128.1 (2C), 126.8, 124.7, 124.2, 120.1, 111.7, 109.5, 102.6, 97.5, 54.6, 54.6, 54.4, 54.1, 47.5 ppm. IR (film, $\mathrm{cm}^{-1}$ ): 2805, 1664, 1587, 1523, 1320, 1104, 897, 816. MS (ESI): $m / z 365(\mathrm{M}+\mathrm{H})^{+}$, HRMS (ESI): $m / z$ calcd for $\mathrm{C}_{23} \mathrm{H}_{25} \mathrm{O}_{3}[\mathrm{M}+\mathrm{H}]^{+}$365.1747, found 365.1756.

((2-Methoxyphenyl)methylene)dibenzene (3ba). Prepared following the general procedure A. Rf: 0.5 (1\% EtOAc in Hex). Yield: $71 \%,{ }^{1} \mathrm{H}$ NMR (400 $\left.\mathrm{MHz}, \mathrm{CDCl}_{3}\right): \delta 7.27-6.79(\mathrm{~m}, 14 \mathrm{H})$, $5.48(\mathrm{~s}, 1 \mathrm{H}), 3.75$ (s, 3H) ppm. ${ }^{13} \mathrm{C} \mathrm{NMR}\left(100 \mathrm{MHz}, \mathrm{CDCl}_{3}\right):$ 158.0, 144.3 (2C), 136.1, 130.4 (2C), 129.4 (5C), 128.3 (4C), 126.29 (2C), 113.7, 56.0, $55.2 \mathrm{ppm}$. IR (film, $\mathrm{cm}^{-1}$ ): 2943, 1685, 1576, 1280, 810, 730. MS (ESI): $m / z 275(\mathrm{M}+\mathrm{H})^{+}$. HRMS (ESI): $m / z$ calcd for $\mathrm{C}_{20} \mathrm{H}_{19} \mathrm{O}[\mathrm{M}+\mathrm{H}]^{+}$275.1430, found 275.1439.

Triphenylmethane (3ca). Prepared following the general procedure A. Rf: 0.5 (1\% EtOAc in Hex). Yield: $67 \%,{ }^{1} \mathrm{H}$ NMR $\left(400 \mathrm{MHz}, \mathrm{CDCl}_{3}\right): \delta 7.33-7.09(\mathrm{~m}, 15 \mathrm{H}), 5.54(\mathrm{~s}, 1 \mathrm{H}) \mathrm{ppm} .{ }^{13} \mathrm{C}$ NMR (100 MHz, $\mathrm{CDCl}_{3}$ ): 143.9 (3C), 129.5, 128.3 (6C), 128.3 (6C), 128.2, 126.3, 56.9 ppm. IR (film, $\mathrm{cm}^{-1}$ ): 2987, 1642, 1586, 1572, 1121. MS (ESI): $m / z 245(\mathrm{M}+\mathrm{H})^{+}$. HRMS (ESI): $\mathrm{m} / z$ calcd for $\mathrm{C}_{19} \mathrm{H}_{17} \mathrm{O}[\mathrm{M}+\mathrm{H}]^{+}$245.1325, found 245.1329.

1-((4-Chlorophenyl)(phenyl)methyl)-2-methoxybenzene (3bi). Prepared following the general procedure A. Rf: 0.3 (2\% EtOAc in Hex). Yield: $62 \% .{ }^{1} \mathrm{H}$ NMR (400 MHz, $\left.\mathrm{CDCl}_{3}\right): \delta 7.25-7.19(\mathrm{~m}, 6 \mathrm{H})$, 7.07-6.99 (m, 4H), 6.87-6.79 (m, 3H), $5.87(\mathrm{~s}, 1 \mathrm{H}), 3.69(\mathrm{~s}$, $3 \mathrm{H}) \mathrm{ppm} .{ }^{13} \mathrm{C} \mathrm{NMR}\left(100 \mathrm{MHz}, \mathrm{CDCl}_{3}\right):$ 157.0, 143.3, 142.5, 132.1, 131.8, 130.7, 130.2, 129.4 (2C), 128.2 (5C), 127.8, 126.2, 120.3, 110.7, 55.5, 49.0 ppm. IR (film, $\mathrm{cm}^{-1}$ ): 2763, 1675, 1297, 981, 824,
747, 615. MS (ESI): $m / z 309(\mathrm{M}+\mathrm{H})^{+}$. HRMS (ESI): $m / z$ calcd for $\mathrm{C}_{20} \mathrm{H}_{18} \mathrm{ClO}[\mathrm{M}+\mathrm{H}]^{+}$309.1041, found 309.1050.

((4-(Trifluoromethyl)phenyl)methylene)dibenzene

(3cj). Prepared following the general procedure A. Rf: 0.5 (1\% EtOAc in Hex). Yield: 56\%. ${ }^{1} \mathrm{H}$ NMR (400 MHz, $\left.\mathrm{CDCl}_{3}\right): \delta 7.54-7.52(\mathrm{~m}$, 2H), 7.31-7.21 (m, 8H), 7.10-7.08 (m, 4H), 5.59 (s, 1H) ppm. ${ }^{13} \mathrm{C}$ NMR (100 MHz, $\mathrm{CDCl}_{3}$ ): 148.0, 142.9 (2C), 129.7, 129.3 (6C), 128.5 (6C), 126.6, 125.2, 125.2, 56.6. IR (film, $\mathrm{cm}^{-1}$ ): 2812, 1664, 1543, 1320, 932, 815. MS (ESI): $m / z 313(\mathrm{M}+\mathrm{H})^{+}$. HRMS (ESI): $m /$ $z$ calcd for $\mathrm{C}_{20} \mathrm{H}_{16} \mathrm{~F}_{3}[\mathrm{M}+\mathrm{H}]^{+}$313.1199, found 313.1198.

4-((2-Fluoro-5-nitrophenyl)(phenyl)methyl)-1,1'-biphenyl (3dk). Prepared following the general procedure B. Rf: 0.5 (3\% EtOAc in Hex). Yield: $42 \%,{ }^{1} \mathrm{H}$ NMR (400 MHz, $\left.\mathrm{CDCl}_{3}\right): \delta 7.58-7.54(\mathrm{~m}, 4 \mathrm{H})$, 7.45-7.32 (m, 6H), 7.29-7.25 (m, 7H), 5.48 (s, 1H) ppm. ${ }^{13} \mathrm{C}$ NMR (100 MHz, $\left.\mathrm{CDCl}_{3}\right): 145.2,142.1,140.8,140.0,137.8,132.3,130.7$, 130.0, 128.9, 128.7, 128.4, 128.3 (4C), 128.1 (2C), 127.6 (2C), 127.3, 127.2, 127.1, 127.0, 126.9, 79.8. IR (film, $\mathrm{cm}^{-1}$ ): 2883, 1904, 1684, 1892, 1543, 871, 810, 742, 613. MS (ESI): $m / z 384(\mathrm{M}+\mathrm{H})^{+}$. HRMS (ESI): $m / z$ calcd for $\mathrm{C}_{25} \mathrm{H}_{19} \mathrm{FNO}_{2}[\mathrm{M}+\mathrm{H}]^{+} 384.1394$, found 384.1402 .

2-((4-Methoxyphenyl)(o-tolyl)methyl)-1,3-dimethylbenzene (3ef). Prepared following the general procedure A. Rf: 0.5 (2\% EtOAc in Hex). Yield: $74 \% .{ }^{1} \mathrm{H}$ NMR (400 $\left.\mathrm{MHz}, \mathrm{CDCl}_{3}\right): \delta 7.42-7.40(\mathrm{~m}$, $1 \mathrm{H}), 7.31-7.12(\mathrm{~m}, 10 \mathrm{H}), 5.40(\mathrm{~s}, 1 \mathrm{H}), 3.38(\mathrm{~s}, 3 \mathrm{H}), 2.26(\mathrm{~s}$, 9H) ppm. ${ }^{13} \mathrm{C}$ NMR (100 $\left.\mathrm{MHz}, \mathrm{CDCl}_{3}\right): 144.6,141.8,141.4$, $141.0,139.9$, 139.6, 136.4, 136.0, 130.6, 130.5, 128.3, 128.2, 127.9, 127.6, 127.4, 126.2, 126.1 (2C), 57.1, 47.4, 19.5 (2C), 19.3 ppm. IR (film, $\mathrm{cm}^{-1}$ ): 2984, 1683, 1317, 981, 823, 728, 604. HRMS (ESI): $m / z$ calcd for $\mathrm{C}_{23} \mathrm{H}_{25} \mathrm{O}[\mathrm{M}+\mathrm{H}]^{+} 317.1900$, found 317.1898 .

4-((2-Nitrophenyl)(phenyl)methyl)-1,1'-biphenyl

(3fk). Prepared following the general procedure A. Rf: 0.4 (2\% EtOAc in Hex). Yield: $49 \%,{ }^{1} \mathrm{H}$ NMR (400 MHz, $\left.\mathrm{CDCl}_{3}\right): \delta 7.58-7.54(\mathrm{~m}$, 5H), 7.46-7.40 (m, 7H), 7.36-7.28 (m, 6H), 5.48 (s, 1H) ppm. IR (film, $\mathrm{cm}^{-1}$ ): 2873, 1796, 1676, 1579, 1544, 923, 815, 794, 745, 631. HRMS (ESI): $m / z$ calcd for $\mathrm{C}_{25} \mathrm{H}_{20} \mathrm{NO}_{2}[\mathrm{M}+\mathrm{H}]^{+} 366.1489$, found 366.1499.

1-((4-Methoxyphenyl)(3-(trifluoromethyl)phenyl)methyl)-3nitrobenzene (3gl). Prepared following the general procedure B. Rf: 0.4 (3\% EtOAc in Hex). Yield: $51 \%$. ${ }^{1} \mathrm{H}$ NMR $(400 \mathrm{MHz}$, $\left.\mathrm{CDCl}_{3}\right): \delta 7.85-7.79(\mathrm{~m}, 1 \mathrm{H}), 7.56-7.51(\mathrm{~m}, 2 \mathrm{H}), 7.42-7.36(\mathrm{~m}$, $3 \mathrm{H}), 7.09-7.06(\mathrm{~m}, 2 \mathrm{H}), 7.01-7.00(\mathrm{~m}, 2 \mathrm{H}), 6.85-6.82(\mathrm{~m}, 2 \mathrm{H})$, 5.85 (s, 1H), 3.80 (S, 3H) ppm. IR (film, $\mathrm{cm}^{-1}$ ): 2907, 1886, 1673, $1584,1543,1281,942,881,634$. HRMS (ESI): $\mathrm{m} / \mathrm{z}$ calcd for $\mathrm{C}_{21} \mathrm{H}_{17} \mathrm{~F}_{3} \mathrm{NO}_{3}[\mathrm{M}+\mathrm{H}]^{+}$388.1155, found 388.1149.

1-Chloro-3-((4-methoxyphenyl)(3-(trifluoromethyl)phenyl)methyl)benzene ( $3 \mathbf{h l})$. Prepared following the general procedure B. Rf: 0.2 (2\% EtOAc in Hex). Yield: $56 \%$. ${ }^{1} \mathrm{H}$ NMR $(400 \mathrm{MHz}$, $\left.\mathrm{CDCl}_{3}\right): \delta 7.24-7.22(\mathrm{~m}, 1 \mathrm{H}), 7.04-6.98(\mathrm{~m}, 2 \mathrm{H}), 6.95-6.93(\mathrm{~m}$, $3 \mathrm{H})$, 6.89-6.88 (m, 2H), 6.85-6.84 (m, 1H), 6.82-6.79 (m, 2H), 6.63-6.62 (m, 1H), $5.82(\mathrm{~s}, 1 \mathrm{H}), 3.78(\mathrm{~S}, 3 \mathrm{H}) \mathrm{ppm}$. IR (film, $\mathrm{cm}^{-1}$ ): 2928, 1691, 1564, 1298, 911, 828, 804. HRMS (ESI): $\mathrm{m} / \mathrm{z}$ calcd for $\mathrm{C}_{21} \mathrm{H}_{17} \mathrm{ClF}_{3} \mathrm{O}[\mathrm{M}+\mathrm{H}]^{+}$377.0915, found 377.0919.

2-Benzhydrylthiophene (5). Prepared following the general procedure A. Rf: 0.6 (1\% EtOAc in Hex). Yield: 50\%. ${ }^{1} \mathrm{H}$ NMR $\left(400 \mathrm{MHz}, \mathrm{CDCl}_{3}\right): \delta 7.30-7.17(\mathrm{~m}, 11 \mathrm{H}), 6.92(\mathrm{~m}, 1 \mathrm{H}), 6.68(\mathrm{~m}$, $1 \mathrm{H}), 5.67$ (s, 1H) ppm. ${ }^{13} \mathrm{C}$ NMR (100 MHz, $\left.\mathrm{CDCl}_{3}\right): 147.9,143.8$ 
(2C), 128.8 (4C), 128.4 (4C), 126.7, 126.6, 126.4 (2C), 124.5, 52.1. IR (film, $\mathrm{cm}^{-1}$ ): 1592, 1585, 1365, 623. MS (ESI): $\mathrm{m} / \mathrm{z} 251(\mathrm{M}+$ $\mathrm{H})^{+}$. HRMS (ESI): $m / z$ calcd for $\mathrm{C}_{17} \mathrm{H}_{15} \mathrm{~S}[\mathrm{M}+\mathrm{H}]^{+} 251.0889$, found 251.0892 .

\section{Conclusion}

In conclusion, we reported an efficient pathway for synthesizing various triarylmethanes using $\mathrm{sp}^{2}-\mathrm{sp}^{3}$ decarboxylative cross coupling reactions. These reactions showed functional group tolerance, providing moderate to good yields of diverse triarylmethanes in less complicated steps. Decarboxylative cross coupling on heteroaryl methyl iodide provides an opportunity for accessing diverse biologically important triarylmethanes and work in this direction is currently underway.

\section{Acknowledgements}

This research project was partly supported by DST project (SB/ S1/OC-93/2013). SLKM and SB thank Council of Scientific and Industrial Research (CSIR), India for research fellowship. Instrumental facilities from SAIF, CDRI is acknowledged (CDRI Communication No. 9408).

\section{Notes and references}

1 (a) S. Mondal and G. Panda, RSC Adv., 2014, 4, 28317-28358; (b) S. K. Das Shagufta and G. Panda, Tetrahedron Lett., 2005, 46, 3097.

2 (a) P. Singh, S. K. Manna, A. K. Jana, T. Saha, P. Mishra, S. Bera, M. K. Parai, M. S. L. Kumar, S. Mondal, P. Trivedi, V. Chaturvedi, S. Singh, S. Sinha and G. Panda, Eur. J. Med. Chem., 2015, 95, 357; (b) R. Palchaudhuri, V. Nesterenko and P. J. Hergenrother, J. Am. Chem. Soc., 2008, 130, 10274; (c) R. S. Dothager, K. S. Putt, B. J. Allen, B. J. Leslie, V. Nesterenko and P. J. Hergenrother, J. Am. Chem. Soc., 2005, 127, 8686-8696; (d) B. A. Ellsworth, W. R. Ewing and E. Jurica, US Pat., 2011/0082165A1, 2011.

3 J. Kim, H. Kim and S. B. Park, J. Am. Chem. Soc., 2014, 136, 14629.

4 (a) M. R. Detty, S. L. Gibson and S. J. Wagner, J. Med. Chem., 2004, 47, 3897; (b) S. K. Das, Shagufta and G. Panda, Tetrahedron, 2006, 62, 6731.

5 (a) C. D. Mason and F. F. Nord, J. Org. Chem., 1951, 16, 722; (b) V. V. Ghaisas, B. J. Kane and F. F. Nord, J. Org. Chem., 1958, 23, 560; (c) M. Irie, J. Am. Chem. Soc., 1983, 105,
2078; (d) R. Muthyala, A. R. Katritzky and X. F. Lan, Dyes Pigm., 1994, 25, 303.

6 (a) D. F. Duxbury, Chem. Rev., 1993, 93, 381; (b) M. S. Shchepinov and V. A. Korshun, Chem. Soc. Rev., 2003, 32, 170.

7 G. Panda, M. K. Parai, S. K. Das Shagufta, M. Sinha, V. Chaturvedi, A. K. Srivastava, Y. S. Manju, A. N. Gaikwad and S. Sinha, Eur. J. Med. Chem., 2007, 42, 410.

8 M. Nambo, Z. T. Ariki, D. Canseco-Gonzalez, D. D. Beattie and C. M. Crudden, Org. Lett., 2016, 18, 2339.

9 (a) G. Pallikonda and M. Chakravarty, J. Org. Chem., 2016, 81, 2135; (b) Y. Z. Li, B. J. Li, X. Lu, S. Lin and Z. J. Shi, Angew. Chem., Int. Ed., 2009, 48, 3817; (c) X. Ji, T. Huang, W. Wu, F. Liang and S. Cao, Org. Lett., 2015, 17, 5096.

10 M. Nambo and C. M. Crudden, ACS Catal., 2015, 5, 4734.

11 T. Niwa, H. Yorimitsu and K. Oshima, Org. Lett., 2007, 9(12), 2373.

12 J. González, C. P. Calleja, L. A. López and R. Vicente, Angew. Chem., Int. Ed., 2013, 52, 5853.

13 Y. Xia, F. Hu, Z. Liu, P. Qu, R. Ge, C. Ma, Y. Zhang and J. Wang, Org. Lett., 2013, 15, 1784.

14 B. L. Taylor, M. R. Harris and E. R. Jarvo, Angew. Chem., Int. Ed. Engl., 2012, 51, 7790.

15 S. Tabuchi, K. Hirano, T. Satoh and M. Miura, J. Org. Chem., 2014, 79, 5401.

16 (a) Y. Luo and J. Wu, Chem. Commun., 2010, 46, 3785; (b) N. Rodriquez and L. J. Goossen, Chem. Soc. Rev., 2011, 40, 5030; (c) J. Zhang, A. Bellomo, N. Trongsiriwat, T. Jia, P. J. Carroll, S. D. Dreher, M. T. Tudge, H. Yin, J. R. Robinson, E. J. Schelter and P. J. Walsh, J. Am. Chem. Soc., 2014, 136, 6276.

17 J. Tang and L. J. Goossen, Org. Lett., 2014, 16, 2664.

18 J. M. Becht, C. Catala, C. L. Drian and A. Wagner, Org. Lett., 2007, 9, 1781.

19 K. Park, G. Bae, J. Moon, J. Choe, K. H. Song and S. Lee, J. Org. Chem., 2010, 75, 6244.

20 (a) W. W. Zhang, X. G. Zhang and J. H. Li, J. Org. Chem., 2010, 75, 5259; (b) F. Bilodeau, M. C. Brochu, N. Guimond, K. H. Thesen and P. Forgione, J. Org. Chem., 2010, 75, 1550.

21 L. J. Goossen, C. Linder and A. Cotte, Saltigo GmbH, DE $102007016971,2007$.

22 B. P. Bandgar, V. S. Sadavarte and L. S. Uppalla, Tetrahedron Lett., 2001, 42, 951.

23 L. J. Goossen, G. Deng and L. M. Levy, Science, 2006, 313, 662. 